\title{
Caracterización de la gestión de la operación del Centro MAC Ventanilla a partir del Modelo EFQM ${ }^{1}$ \\ Characterization of the operation management of the MAC Ventanilla Center based on EFQM Model
}

\author{
Gabriel Marcial Avilés Elias \\ Pontificia Universidad Católica del Perú \\ gabriel.aviles@pucp.pe \\ Allison Andrea Moreno Herrera \\ Pontificia Universidad Católica del Perú \\ allison.moreno@pucp.pe
}
1. El presente artículo se basa en la tesis de licenciatura Caracterización del proceso de operacionalización del Centro MAC Ventanilla a partir del Modelo EFQM en el periodo 2018-2019, presentada ante la Facultad de Gestión de la Pontificia Universidad Católica del Perú (Aviles \& Moreno, 2019).


En Perú, en el marco de la Política Nacional de Modernización de la Gestión Pública, se viene trabajando por un Estado orientado a los ciudadanos y con entidades públicas que funcionen articuladamente. Por ello, se crea la Plataforma de Mejor Atención al Ciudadano (Plataforma MAC) para mejorar la calidad de los servicios del Estado, articulándolos y acercándolos a los ciudadanos mediante diversos canales de atención. En 2014, el Centro MAC Ventanilla (CMV) inicia su funcionamiento gestionado por la Municipalidad Distrital de Ventanilla, logrando congregar 11 entidades públicas y realizar 522488 atenciones durante el año 2018.

El presente artículo describe y analiza la gestión de la operación del CMV mediante los agentes facilitadores del Modelo EFOM de Excelencia. Si bien el CMV puede constituirse como uno de los principales canales presenciales debido a su alto número de atenciones, aún queda pendiente identificar oportunidades de mejora, establecer prioridades e innovar.

Palabras clave: modernización de la gestión pública, calidad, plataforma MAC, servicios integrados, Modelo EFQM de Excelencia

In Peru, within the framework of the National Policy for the Modernization of Public Management, work have been carried out for a State oriented towards citizens and with public entities that operate in an articulated manner. For this reason, the Best Citizen Service Platform (MAC Platform, for its initials in Spanish) is created to improve the quality of State services, articulating and bringing them closer to citizens through various customer service channels. In 2014, the MAC Ventanilla Center (CMV) began its operation managed by the Ventanilla District Municipality, achieving to gather 11 public entities and carry out 522488 services during the year 2018.

This article describes and analyzes the management of the CMV operation through the enablers of the EFQM Excellence Model. Although the CMV can become one of the main face-to-face channels due to its high number of services, it is still pending to identify opportunities for improvement, establish priorities and innovate.

Keywords: modernization of public management, quality, MAC Platform, integrated services, EFQM Excellence Model 


\section{Introducción}

Perú está en un proceso de modernización de la gestión pública que responde al bajo desempeño del Estado, reflejado en los bajos niveles de satisfacción ciudadana (Decreto Supremo N 004-2013-PCM), lo cual, de acuerdo a González (2009), puede llevar al Estado a un escenario de deslegitimación y, asimismo, debilita el contrato social, entendido como un pacto tácito entre el Estado y los ciudadanos (OCDE et al., 2018). Como evidencia de ello, se puede mencionar que la encuesta de Ipsos Perú de 2017 reveló que el 29 \% de peruanos encuestados no se sentía satisfecho con su visita a las entidades públicas evaluadas y que el $40 \%$ de los usuarios de dichas entidades desconfiaba del Estado.

En el 2002 se promulgó la Ley N²7658, Ley Marco de Modernización de la Gestión del Estado, declarando al Estado peruano en proceso de modernización con la finalidad de obtener mayores niveles de eficiencia en el aparato estatal y, en consecuencia, una mejor atención a la ciudadanía. En este contexto, la Presidencia del Consejo de Ministros (PCM), ente rector del proceso de modernización de la gestión pública, ha venido liderando la Política Nacional de Modernización de la Gestión Pública al 2021 desde su aprobación en 2013, en la cual se dispone la elaboración del Plan Nacional de Simplificación Administrativa. Dicho plan fue finalmente aprobado con el objetivo de mejorar la calidad, eficiencia y oportunidad de los procedimientos y servicios administrativos que la ciudadanía realiza ante la administración pública (Resolución Ministerial Nº48-2013-PCM); y planteó la implementación de los centros MAC y otras acciones con el fin de mejorar el nivel de atención a la ciudadanía.

La Plataforma MAC, anteriormente identificada como Estrategia MAC, se crea con la finalidad de optimizar el tiempo y los costos involucrados en la interacción de los ciudadanos con las entidades del Estado mediante diversos canales de atención, destinados a integrar diversas entidades públicas (Contreras, 2015). Los centros MAC, el canal presencial de la Plataforma MAC, son un componente fundamental de este mecanismo. Para poder implementarlos la PCM estableció inicialmente, mediante el Decreto Supremo Nº55-2018-PCM, que estos primero podrían ser establecidos por la PCM, asociándose únicamente con las entidades públicas que brindarán sus servicios, o delegando posteriormente la operación y el mantenimiento a una entidad aliada (del Gobierno regional, municipal o una entidad privada); y segundo, que podrían implementarse a través de una entidad aliada, la cual luego podría encargarse o no de la operación y el mantenimiento.

En ese sentido, en el año 2014 se inauguró el Centro MAC Ventanilla (CMV, en adelante) en coordinación con la Municipalidad de Ventanilla (MDV, en adelante), siendo esta última entidad la encargada de implementar, operar y mantener el CMV, lo que la convierte en una entidad aliada de la PCM. Al 2018, el CMV contaba con 12 entidades (11 entidades públicas y una privada) participantes, las cuales brindan orientación y prestan servicios de trámites administrativos, realizando 2077210 atenciones desde su implementación, con un promedio de atenciones 43541 mensuales.

El modelo de funcionamiento de los centros MAC, establecido mediante la Resolución de Secretaría de Gestión Pública № 005-2019-PCM-SGP, se sustenta en los procesos, la estructura orgánica que los soporta y los resultados. Respecto a lo primero, el CMV organiza su funcionamiento sobre cuatro procesos clave que coadyuvan a incorporar 
un ciclo de mejora continua en las operaciones del CMV: gestión y control, gestión de la operación, gestión y evaluación de la prestación de los servicios, y gestión de recursos e infraestructura. El primero de ellos, la gestión y el control del centro MAC, comprende las actividades que permiten asegurar la calidad de los procesos; mientras que la gestión de la operación agrupa las actividades que tienen por objeto garantizar un servicio eficiente. Por otro lado, la gestión y evaluación de la prestación de los servicios comprende actividades relacionadas con el personal y el cumplimiento del perfil idóneo para cada puesto; y la gestión de recursos e infraestructura, a su vez, dota de recursos físicos y tecnológicos, infraestructura y servicios necesarios a todos los procesos anteriores (SGP-PCM, 2019).

El funcionamiento de los centros MAC contiene una lógica de procesos subyacente, lo cual implica que los procesos claves y sus procedimientos se encuentran interrelacionados (figura 1); es decir, "el resultado de la ejecución de un procedimiento es la entrada necesaria para el correcto funcionamiento de otro procedimiento» (SGP-PCM, 2019).

Figura 1. Interrelación de procesos del centro MAC

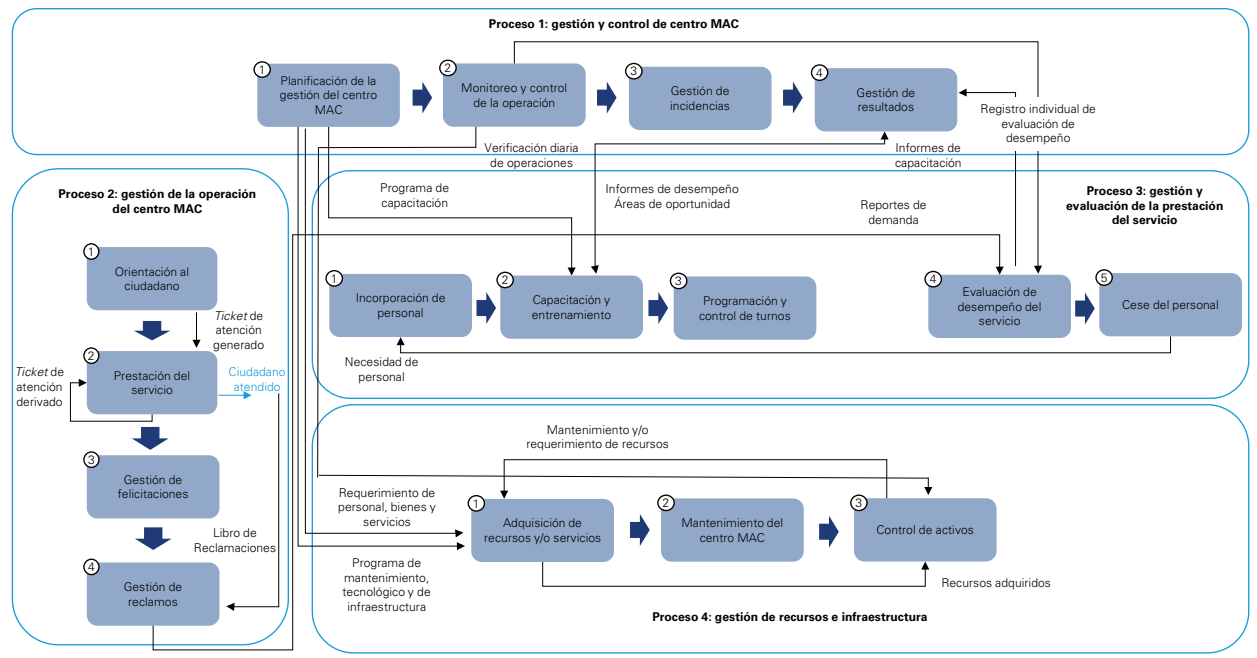

Fuente: adaptado de SGP-PCM (2019).

En la investigación de Prieto (2013) se mencionan factores negativos que pueden identificarse en la implementación de la Plataforma MAC, tales como la inexperiencia en el uso de soluciones de innovación, la alta rotación de funcionarios y problemas en el intercambio de información entre instituciones, lo cual se pudo evidenciar en el CMV a partir de entrevistas exploratorias. En esta línea, es difícil corregir dichos fallos cuando se implementa un centro MAC, puesto que no existe una evaluación de los mismos que permita conocer la situación actual del canal presencial de la Plataforma MAC. Esta ausencia dificulta la identificación y puesta en marcha de mejoras, pues los fallos durante la implementación repercuten en la operación de un centro MAC, dificultando obtener mejores niveles de satisfacción ciudadana. 
En ese sentido, el objetivo de esta investigación es caracterizar la gestión de la operación a través de un estudio de caso único del CMV para el periodo 2018-2019. Para ello, se emplearán los componentes contemplados en el Modelo EFQM de Excelencia debido a que provee un entendimiento integral y holístico de los factores vinculados a la gestión de la operación de una organización. Si bien el modelo de funcionamiento de los centros MAC es ad hoc, la metodología utilizada en este estudio podría servir de referencia para casos similares o para la misma tipología de centros MAC. Asimismo, la investigación permitirá que el CMV, la MDV o la Secretaría de Gestión Pública de la PCM (en adelante, SGP-PCM) identifiquen oportunidades de mejora, establezcan prioridades y lleven a cabo innovaciones.

La estructura de este artículo es la siguiente: primero, un marco teórico que aborde los procesos de reforma del Estado, la importancia de la calidad en la Administración Pública y el Modelo EFQM. Después, se presenta la metodología para el estudio del CMV, para luego seguir con los hallazgos obtenidos. Finalmente, se plantean discusiones en torno a los hallazgos y conclusiones del estudio.

\section{Marco teórico}

\subsection{La reforma de la Administración Pública}

El Estado ha transitado por distintas reformas que han generado cambios en la manera como se gestiona la Administración Pública. Según Montoya (2010), la reforma del Estado representa la necesidad de reajustar el aparato estatal con la finalidad de que los intereses de la sociedad sean atendidos ante la percepción de insatisfacción frente a la resolución de sus demandas. Robinson (2015) y Bresser Pereira (2004), por su parte, identifican tres modelos principales de Administración Pública característicos del siglo XX y de la actualidad: la Administración de carácter burocrático, la nueva gestión pública y la nueva gobernanza pública.

El modelo de la Administración Pública de carácter burocrático estuvo basado en los postulados de Weber (1987), los cuales concebían la Administración como un sistema de reglas racionalizado, jerárquico y centrado (citado en Alza, 2012). Debido al gran tamaño y complejidad que alcanzó, se presentaron dificultades para atender las necesidades de los ciudadanos. Es así como a inicios de 1980 surge la nueva gestión pública, que recoge conceptos como el gerencialismo; es decir, la búsqueda del restablecimiento de los principios gerenciales de dirección de actividades empresariales (Aucoin, 1996, citado en Olías de Lima, 2001). En ese contexto, las actividades se enfocaron en los productos y servicios más adecuados para los clientes, así como en la calidad y el valor que generaban; y se le brindó mayor importancia a la creación de una cultura organizativa, el compromiso con la calidad, la mejora de procesos y la innovación, entre otros elementos (Barzelay, 2003).

A pesar de ello, la nueva gestión pública redujo la complejidad del Estado a niveles que ocasionaron su fragmentación. En ese contexto, la nueva gobernanza pública surge como una aproximación a la búsqueda de coordinación entre instituciones de la sociedad con la finalidad de lograr objetivos colectivos. Más recientemente, este enfoque ha planteado cambios como la importancia de la tecnología, la cual, de acuerdo a Dunleavy (2005), ha 
colaborado a la reintegración de las unidades estatales surgidas a raíz de la fragmentación, la creación de una Administración más holística y la digitalización de las operaciones. Asimismo, ha traído a la discusión nuevos conceptos como el Whole of Government, el cual es una mirada que evidencia el deseo de llevar a cabo iniciativas de coordinación horizontal y vertical a fin de utilizar mejor los recursos, creando sinergias entre los actores para así optimizar el acceso a los servicios públicos (Christensen \& Laegreld, 2007).

Finalmente, cabe señalar que las reformas mencionadas no se desvinculan totalmente dentro de la organización pública pues, tal como lo señalan Patapas et al. (2014), es de esta manera como los sistemas existentes se han desarrollado y mejorado. En ese sentido, es importante rescatar que la Administración Pública continúa siendo un sistema jerárquico de reglas, el cual ha recogido conceptos como la calidad y el valor que generan productos y servicios adecuados sobre los usuarios; y que esta finalmente se está aproximando a un enfoque que busca mayor coordinación entre instituciones a fin de brindar mejores servicios públicos a los ciudadanos.

\subsection{La calidad en la Administración Pública}

Según Camarasa (2004), las iniciativas de reformas en el Estado están relacionadas a la insatisfacción ciudadana por servicios públicos. Tal como lo señala el Centro Latinoamericano de Administración para el Desarrollo (CLAD, 2008), el principal reto en la gestión pública referido a la calidad es su orientación al servicio de las necesidades y expectativas ciudadanas, así como su compromiso con alcanzar una sociedad de bienestar. Como se observa, la Administración Pública ha experimentado procesos de adaptación en distintos momentos, planteando nuevos mecanismos para atender satisfactoriamente a los ciudadanos. Uno de los enfoques a rescatar para dicho objetivo es el uso de la calidad, el cual busca obtener mejores resultados y niveles de desempeño dentro de la Administración Pública.

El enfoque de la calidad ha evolucionado hacia el desarrollo de visiones más proactivas y sistémicas que plantean crear valor a partir de elementos como la cultura organizacional, la prestación de servicios y la reingeniería de procesos, así como enfoques globales como la gestión total de la calidad, la cual toma en cuenta las necesidades de los consumidores para el diseño de la oferta de bienes y servicios (Camisón et al., 2006; Cubillo \& Rozo, 2017). Dicho enfoque es relevante en tanto gestionar la calidad en las organizaciones públicas implica garantizar derechos y el desarrollo de los ciudadanos.

De acuerdo a Camarasa (2004), las estrategias enfocadas a la mejora de la calidad de los servicios públicos deben tener dos prioridades: reflexionar sobre los principales espacios dentro de la Administración Pública sobre los que se debe actuar e introducir técnicas de gestión para un funcionamiento más eficiente, eficaz, rápido y próximo a los ciudadanos. En ese sentido, los modelos de evaluación de la calidad se presentan como una herramienta favorable para dicho fin, puesto que surgieron estableciendo nuevos patrones y estándares de calidad.

Los modelos de calidad contienen una base y columna de evaluación holística de la calidad que toma en cuenta una serie de parámetros nacionales o internacionales, y no únicamente el servicio en sí mismo, lo que permite identificar las oportunidades de 
mejora (Vásquez, 2014). En similar dirección, Torrubiano et al. (2011) mencionan que los modelos de excelencia de la calidad están fundados sobre los principios de excelencia de las organizaciones y plantean así una secuencia metodológica para el logro de resultados satisfactorios. A lo largo de los años, estos modelos cobraron relevancia en distintas organizaciones, de tal modo que fueron también adaptados y considerados en las del sector público.

\subsection{El Modelo EFQM de Excelencia}

Uno de los principales modelos de excelencia es el Modelo EFQM, el cual, desde su origen en el año 1988, se sustentó en conceptos esenciales con enfoque en la eficiencia y perspectiva de mejora continua que permitirían a la organización alcanzar la excelencia (Saiz \& Olalla, 2013). Tal es así que el Ministerio de Administraciones Públicas de España impulsó su aplicación como referente continental adecuado para organizaciones de todo tipo, independientemente de su sector y tamaño. En el año 1999 se puso a disposición de los gestores públicos un instrumento de autoevaluación para sus organizaciones (la Guía de Autoevaluación para la Administración Pública), preparado a partir de los principios esenciales del Modelo EFQM (Vásquez, 2014).

Este modelo es un marco de trabajo no prescriptivo compuesto por nueve criterios agrupados en agentes facilitadores y resultados (European Foundation for Quality Management, 2012). El primer grupo está compuesto por el liderazgo, las personas, la estrategia, las alianzas y recursos, y los procesos, los cuales se refieren a la gestión interna de la organización; el segundo, por los resultados en las personas, en la sociedad, en los usuarios y por resultados claves, los cuales tratan sobre los efectos de dicha gestión. Esta metodología permite fomentar el aprendizaje, la creatividad y la innovación (figura 2). Por tanto, a través del conocimiento del rendimiento de la organización, el Modelo EFOM permite comprender su estado actual y orientar las acciones en concordancia con los principios de la gestión de la calidad.

Figura 2. Modelo EFQM de Excelencia

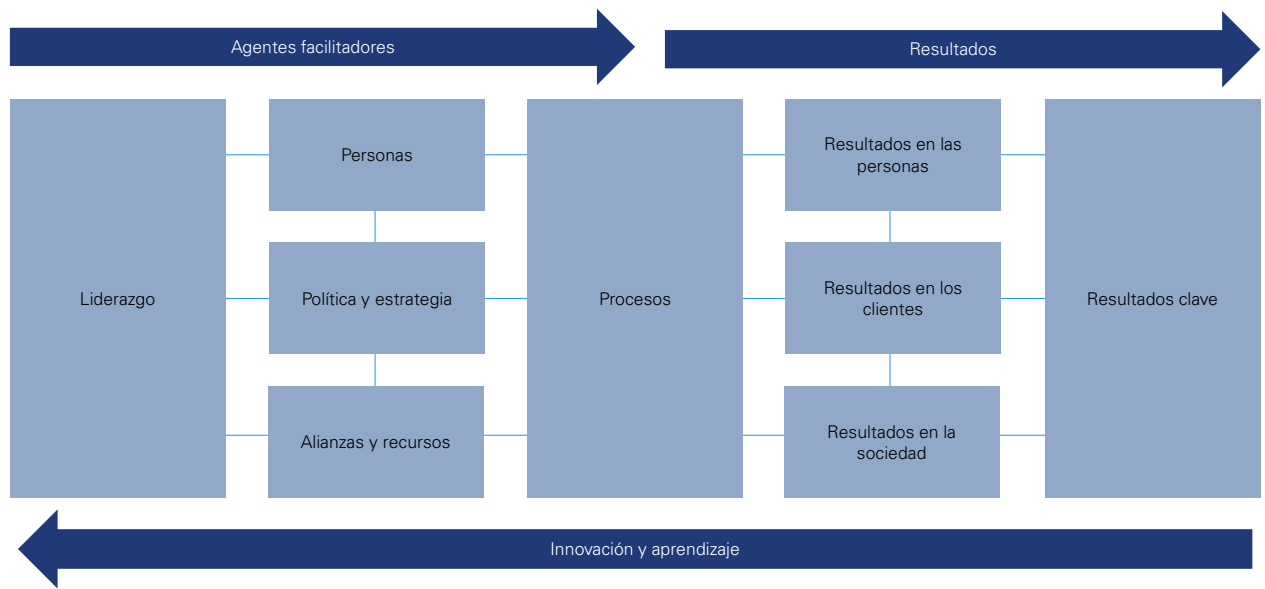

Fuente: Junta de Andalucía (s/f). 
Para el presente estudio, se ha optado por la aplicación del Modelo EFQM por presentarse como un modelo holístico y de cómoda adaptabilidad a organizaciones de diversos sectores. El Modelo EFQM es una herramienta poderosa de diagnóstico que permite identificar fortalezas y oportunidades de mejora que no hubieran sido evidentes de otro modo (Porter \& Taner, 1998, citados en Jacobs \& Suckling, 2007), y su aplicación resulta en una evaluación que se presenta como insumo para el establecimiento de un modelo de gestión de la calidad total (George et al., 2003, citado en Ruiz et al., 2016).

Aunque la Plataforma MAC incorpora el componente de calidad basándose en la Norma ISO 9001:2008, esta es aún una perspectiva incompleta e insuficiente para configurar un servicio de calidad que satisfaga todas las necesidades de los usuarios. En este contexto, el diagnóstico que facilita el Modelo EFOM admite diferentes niveles de excelencia, ya que puede iniciarse por el control de calidad — fase actual del CMV, con el cumplimiento de estándares propuestos por la mencionada Norma ISO_, seguir por el aseguramiento y terminar por la gestión de la calidad total (Muñoz, 1999, citado en Ruiz et al., 2016). Por lo tanto, la aproximación inicial a la operación del CMV a través de la aplicación del Modelo EFQM brindaría futuros beneficios como la maximización de la calidad, la optimización del uso de recursos productivos de bienes y servicios, y el aumento del grado de satisfacción de quienes interactúan con el centro MAC (Saiz \& Olalla, 2013).

Finalmente, la presente investigación se concentra sobre los agentes facilitadores del Modelo EFOM, ya que se ha determinado que la actuación de la organización sobre estos tiene una fuerte influencia en los resultados (Tejedor et al., 2011). Asimismo, Ruiz et al. (2016) seleccionan los agentes facilitadores para el estudio de un organismo autónomo de España ante la ausencia de un modelo de gestión de la calidad.

\section{Metodología}

Con respecto a la metodología, el alcance de la investigación es descriptivo, puesto que tiene como objetivo especificar las características de la gestión interna del CMV con base en los agentes facilitadores del Modelo EFOM. En ese sentido, se ha planteado un enfoque cualitativo, empleando una estrategia de estudio de caso único holístico que aborda los procesos de los centros MAC. Se optó por un estudio de caso pues estos permiten el uso de diversas técnicas para la recolección de datos, como las entrevistas, el análisis de documentos, los cuestionarios y la observación (Saunders et al., 2009). Siguiendo esa línea, el presente estudio de caso hará uso del método mixto para la recolección de información, el cual consiste en emplear herramientas de corte cualitativo y cuantitativo con dicho fin. Por último, cabe indicar que el horizonte temporal del estudio es transversal, puesto que se está proporcionando información correspondiente al momento de su realización: el periodo 2018-2019 (Ponce \& Pasco, 2015).

\subsection{Secuencia metodológica}

En un primer momento, se hizo una revisión de la literatura y se desarrollaron entrevistas exploratorias, las cuales evidenciaron debilidades en la operación del CMV. Posteriormente, se llevaron a cabo entrevistas semiestructuradas a los responsables de la Plataforma MAC 
y el CMV (funcionarios de la Subsecreataría de Calidad en Atención al Ciudadano de la SGPPCM, la coordinadora del CMV y el especialista de calidad y procesos del CMV). Asimismo, se aplicaron encuestas a 49 personas que formaban parte del personal del CMV (asesores de servicio, orientadores y anfitriones).

Cabe señalar que, debido a que las variables a analizar son no observables, se realizó un análisis de fiabilidad mediante el coeficiente alfa de Cronbach, el cual arrojó valores superiores a 0,8 para todas ellas. Por otro lado, para el análisis factorial, la prueba de esfericidad de Bartlett arrojó el valor de significatividad de 0,000 y la prueba Kaiser-MeyerOlkin determinó el valor de 0,187, denotando alta correlación entre variables. Finalmente, se llevó a cabo un proceso de validación con expertos en el ámbito de la gestión pública, el desarrollo de políticas públicas y la gestión por procesos del sector público con el fin de discutir y recopilar opiniones alrededor de los hallazgos obtenidos.

La información recolectada mediante las entrevistas y encuestas fue codificada respecto a las variables y subvariables de estudio con el uso de programas como Microsoft Excel y ATLAS.ti 7.5. De este modo, con la información ya organizada, se realizó el análisis en torno a las variables, lo que permitió plantear los hallazgos.

\subsection{Operacionalización de variables}

Se ha establecido a los agentes facilitadores del Modelo EFQM de Excelencia como las variables de la investigación. A partir de estas, se definieron 61 subvariables, las cuales han sido elaboradas a partir de las dimensiones de cada agente facilitador del Modelo EFQM, tomando como referencia el Cuestionario de Evaluación de la Junta de Andalucía (s/f).

Tabla 1. Agentes facilitadores, dimensiones y subvariables

\begin{tabular}{|c|c|c|}
\hline Variable & Dimensiones & Subvariables \\
\hline \multirow[t]{14}{*}{ Liderazgo } & \multirow{5}{*}{$\begin{array}{l}\text { Primera dimensión } \\
\text { Los líderes desarrollan la misión y visión, } \\
\text { además de los valores y principios éticos, y } \\
\text { actúan como modelo de referencia dentro de } \\
\text { una cultura de excelencia }\end{array}$} & Desarrollo y comunicación de la misión y visión \\
\hline & & $\begin{array}{l}\text { Desarrollo y comunicación de los valores y la } \\
\text { cultura }\end{array}$ \\
\hline & & Estímulo a la autonomía y el trabajo en equipo \\
\hline & & Estímulo a la innovación \\
\hline & & Estímulo al compromiso con la calidad \\
\hline & \multirow{5}{*}{$\begin{array}{l}\text { Segunda dimensión } \\
\text { Los líderes se implican personalmente para } \\
\text { garantizar el desarrollo, la implantación y } \\
\text { mejora continua del sistema de gestión de la } \\
\text { organización }\end{array}$} & Participación para las mejoras \\
\hline & & Motivación para las mejoras \\
\hline & & Coordinación para las mejoras \\
\hline & & Adaptabilidad de la estructura organizacional \\
\hline & & Respuesta a los objetivos y las estrategias \\
\hline & \multirow{2}{*}{$\begin{array}{l}\text { Tercera dimensión } \\
\text { Los líderes se implican con clientes, partners y } \\
\text { representantes de la sociedad }\end{array}$} & Atención de necesidades \\
\hline & & Medios para la atención de necesidades \\
\hline & \multirow{2}{*}{$\begin{array}{l}\text { Cuarta dimensión } \\
\text { Los líderes definen e impulsan el cambio en la } \\
\text { organización }\end{array}$} & Comunicación de los cambios \\
\hline & & Medios para la comunicación de los cambios \\
\hline
\end{tabular}




\begin{tabular}{|c|c|c|}
\hline \multirow[t]{12}{*}{ Estrategia } & \multirow{3}{*}{$\begin{array}{l}\text { Primera dimensión } \\
\text { La estrategia se basa en las necesidades y } \\
\text { expectativas actuales y futuras de los grupos } \\
\text { de interés }\end{array}$} & Información para la implementación \\
\hline & & Información para la operación \\
\hline & & Medios para la comprensión del entorno \\
\hline & \multirow{3}{*}{$\begin{array}{l}\text { Segunda dimensión } \\
\text { La estrategia se basa en la información de los } \\
\text { indicadores de rendimiento, la investigación, el } \\
\text { aprendizaje y las actividades externas }\end{array}$} & Información del rendimiento \\
\hline & & Mecanismos de aprendizaje \\
\hline & & Experiencias externas \\
\hline & \multirow{2}{*}{$\begin{array}{l}\text { Tercera dimensión } \\
\text { La estrategia se desarrolla, revisa y actualiza }\end{array}$} & Alineación de la estrategia \\
\hline & & Modificación de la estrategia \\
\hline & \multirow{4}{*}{$\begin{array}{l}\text { Cuarta dimensión } \\
\text { La estrategia se comunica y despliega median- } \\
\text { te un esquema de procesos clave }\end{array}$} & Identificación de procesos clave \\
\hline & & Reconocimiento de procesos clave \\
\hline & & Comunicación de la estrategia \\
\hline & & Aplicación de procesos clave \\
\hline \multirow[t]{12}{*}{ Personas } & $\begin{array}{l}\text { Primera dimensión } \\
\text { Planificación, gestión y mejora de los recursos } \\
\text { humanos }\end{array}$ & Plan de gestión de personas \\
\hline & \multirow{4}{*}{$\begin{array}{l}\text { Segunda dimensión } \\
\text { Identificación, desarrollo y mantenimiento del } \\
\text { conocimiento y la capacidad de las personas }\end{array}$} & Actividades de desarrollo \\
\hline & & Perfil del personal \\
\hline & & Formación del personal \\
\hline & & Desarrollo profesional \\
\hline & \multirow{2}{*}{$\begin{array}{l}\text { Tercera dimensión } \\
\text { Implicación y asunción de responsabilidades } \\
\text { por parte de las personas de la organización }\end{array}$} & Objetivos del personal \\
\hline & & Percepción sobre los objetivos \\
\hline & \multirow{3}{*}{$\begin{array}{l}\text { Cuarta dimensión } \\
\text { Existencia de un diálogo entre las personas y } \\
\text { la organización }\end{array}$} & Efectividad de la comunicación \\
\hline & & Canales de comunicación \\
\hline & & Disposición de la información \\
\hline & \multirow{2}{*}{$\begin{array}{l}\text { Quinta dimensión } \\
\text { Recompensa y reconocimiento, y atención a } \\
\text { las personas de la organización }\end{array}$} & $\begin{array}{l}\text { Percepción sobre el reconocimiento y la } \\
\text { recompensa }\end{array}$ \\
\hline & & Sistemas de reconocimiento y recompensa \\
\hline \multirow{10}{*}{$\begin{array}{l}\text { Alianzas y } \\
\text { recursos }\end{array}$} & \multirow{2}{*}{$\begin{array}{l}\text { Primera dimensión } \\
\text { Gestión de las alianzas externas }\end{array}$} & Alianzas \\
\hline & & Resultados en el servicio \\
\hline & \multirow{2}{*}{$\begin{array}{l}\text { Segunda dimensión } \\
\text { Gestión de los recursos económicos y } \\
\text { financieros }\end{array}$} & Gestión de los recursos económicos \\
\hline & & Necesidades futuras \\
\hline & $\begin{array}{l}\text { Tercera dimensión } \\
\text { Gestión de los edificios, equipos y materiales }\end{array}$ & Mantenimiento y mobiliario \\
\hline & \multirow{2}{*}{$\begin{array}{l}\text { Cuarta dimensión } \\
\text { Gestión de la tecnología }\end{array}$} & Identificación de nuevas tecnologías \\
\hline & & Percepción de nuevas tecnologías \\
\hline & \multirow{3}{*}{$\begin{array}{l}\text { Quinta dimensión } \\
\text { Gestión de la información y el conocimiento }\end{array}$} & Acceso a información oportuna \\
\hline & & Medios de acceso a la información \\
\hline & & Seguridad de la información \\
\hline
\end{tabular}




\begin{tabular}{|c|c|c|}
\hline \multirow[t]{13}{*}{ Procesos } & \multirow{6}{*}{$\begin{array}{l}\text { Primera dimensión } \\
\text { Diseño y gestión sistemática de los procesos }\end{array}$} & Efectividad del diseño de los procesos \\
\hline & & Satisfacción de necesidades mediante procesos \\
\hline & & Diseño del sistema de gestión por procesos \\
\hline & & $\begin{array}{l}\text { Implementación del sistema de gestión por } \\
\text { procesos y de calidad }\end{array}$ \\
\hline & & Diseño de sistemas de seguimiento y medición \\
\hline & & $\begin{array}{l}\text { Implementación del sistema de seguimiento y } \\
\text { medición }\end{array}$ \\
\hline & \multirow{3}{*}{$\begin{array}{l}\text { Segunda dimensión } \\
\text { Introducción de las mejoras necesarias en los } \\
\text { procesos }\end{array}$} & $\begin{array}{l}\text { Procedimiento para la identificación de oportuni- } \\
\text { dades de mejora }\end{array}$ \\
\hline & & Identificación de oportunidades de mejora \\
\hline & & Comunicación de cambios en los procesos \\
\hline & \multirow{2}{*}{$\begin{array}{l}\text { Tercera dimensión } \\
\text { Diseño y desarrollo de los productos y servi- } \\
\text { cios basándose en las necesidades y expectati- } \\
\text { vas de los clientes }\end{array}$} & Impacto de los procesos \\
\hline & & Protocolos de atención \\
\hline & \multirow{2}{*}{$\begin{array}{l}\text { Cuarta dimensión } \\
\text { Gestión y mejora de las relaciones con los } \\
\text { clientes }\end{array}$} & $\begin{array}{l}\text { Recopilación de sugerencias, reclamos y } \\
\text { encuestas }\end{array}$ \\
\hline & & Sugerencias, reclamos y encuestas \\
\hline
\end{tabular}

Fuente: adaptado de Junta de Andalucía (s/f).

\section{Resultados}

\subsection{Liderazgo}

Se ha determinado que no hay un claro desarrollo de la misión y visión, puesto que estas aún se encontrarían en desarrollo. Sin perjuicio de ello, en el CMV se identificó un conocimiento de la finalidad de los centros MAC por parte de los responsables, así como su comunicación hacia el personal; sin embargo, cabe indicar que una parte de ellos señaló que la misión y visión no les fueron comunicadas. Del mismo modo, con respecto a los valores y la cultura, si bien estos no se encuentran desarrollados de manera clara, sí se observó la presencia de una cultura orientada a la búsqueda de mejoras en la atención al ciudadano, prestando servicios de manera rápida y de forma óptima.

Los hallazgos respecto a una percepción positiva por parte del personal sobre el estímulo al compromiso con la calidad podrían estar relacionados a que los responsables del CMV actúan como referentes al comprender la importancia de la atención adecuada. Por otro lado, también existe la percepción de un sector del personal del CMV que considera que aquellos que lo lideran confían en sus capacidades para la atención ciudadana, lo cual podría explicar cierta preferencia por la autonomía en el accionar del personal frente al trabajo en equipo.

Luego, con respecto al involucramiento de los líderes en el desarrollo y las mejoras del sistema de la organización, este existe tanto desde el CMV como desde la SGP-PCM en torno al proceso de mejoras, ya sea para liderar este aspecto o para tomar la iniciativa y realizar las coordinaciones necesarias. Igualmente, se identificó que la definición de la estructura organizacional de los centros MAC está definida por la SGPPCM y las adaptaciones que a esta se le aplican; por ejemplo, como sucede en el caso 
del CMV, que permanece con los puestos de anfitriones y de especialista de calidad y procesos, estos deben contar con su aprobación. A pesar de lo mencionado, el estímulo a la innovación hacia el personal del CMV y su participación en el planteamiento de mejoras no sería suficiente, ya que no se ha demostrado que este en su mayoría se encuentre de acuerdo con lo primero. Además, a pesar de que las coordinaciones para las mejoras se realizan principalmente en reuniones a nivel estratégico y gerencial, existe disposición para participar del planteamiento de mejoras.

Luego, se identificó que el involucramiento de los líderes para atender y anticipar las necesidades y expectativas de los grupos de interés se da principalmente con los ciudadanos, puesto que consideran importante entender qué es lo que la ciudadanía necesita y ayudar a que esta lo consiga de manera clara y eficaz. Respecto al personal del CMV, este señaló estar mayoritariamente de acuerdo en que la coordinadora del CMV es accesible con ellos, escuchándolos de manera activa y respondiendo a sus necesidades. A pesar de esto, no se observa claramente el involucramiento con actores como las entidades participantes ni entre las entidades responsables (SGP-PCM y CMV), dado que estas se limitarían a atender las necesidades de materiales de oficina en el caso de las primeras y al cumplimiento de los objetivos del servicio en el de las segundas; incluso, habría necesidades que el CMV aún considera desatendidas, como las de espacio y personal.

Finalmente, respecto a la comunicación de los cambios por parte de los líderes, estos se dan principalmente desde un nivel estratégico y gerencial; es decir, se comunican desde la SGP-PCM hacia el equipo responsable del CMV, ya sea a través de reuniones o por medios de comunicación formales. Sin embargo, una parte importante del personal del CMV (33 \%) no presentó una postura definida respecto a si los cambios y sus razones les son comunicados. En ese sentido, se podría advertir que la comunicación hacia el personal en relación a este aspecto no sería suficiente.

\subsection{Estrategia}

Respecto a la estrategia del CMV, esta se relaciona en torno a aquella que se construye para la Plataforma MAC, la cual se ha venido actualizando y desarrollando a través del tiempo mediante los distintos documentos de gestión y/o normativos aprobados desde la SGP-PCM. La estrategia hacia la mejor atención ciudadana se relaciona de manera coherente con las misiones y visiones de las entidades que confluyen en el CMV, ya que estas están definidas de tal manera que apuntan a brindar servicios que beneficien al ciudadano. Por ejemplo, en el caso específico de la PCM y la MDV, ambas buscan brindar servicios de calidad y denotan un enfoque hacia el ciudadano.

Respecto a la implementación del CMV, se observa que se ha identificado el potencial y la idoneidad del distrito de Ventanilla, pues se determinó que sus operaciones benefician a alrededor de 260000 ciudadanos del distrito y de distritos cercanos (Municipalidad Distrital de Ventanilla, s/f). A pesar de ello, se han reconocido medios limitados para comprender el entorno externo. Para ello, se utiliza principalmente el sistema de colas y las encuestas se aplican a los usuarios para recopilar información relacionada al rendimiento del CMV (tiempos de espera, servicios más demandados), así como las 
opiniones de los ciudadanos. Cabe precisar que no se cuenta con mecanismos para conocer mejor el perfil de los ciudadanos que asisten al CMV.

Luego, en el año 2019, la Subsecretaría de Calidad en Atención al Ciudadano (en adelante, SSCAC) de la SGP-PCM detalló una serie de nuevos indicadores por procesos en el Manual de funcionamiento más reciente. Se identificó que el CMV recopila información sobre la cantidad promedio de atenciones y los tiempos de atención, la cual se relaciona con algunos de los indicadores como, por ejemplo, la tasa de atención de citas, el tiempo promedio de espera del ciudadano por trámite o la tasa de reclamos.

Por otro lado, en relación a la recopilación de experiencias externas, si bien la SGP-PCM tomó experiencias internacionales para el desarrollo de la Plataforma MAC, el CMV no recoge experiencias de otros centros MAC a nivel nacional. No obstante, tal como lo señala el especialista de calidad y procesos, ello permitiría que este pueda conocer, por ejemplo, cómo funcionan las operaciones en otros centros MAC con mayor número de atenciones, permitiendo identificar mejoras oportunas ante el aumento de la demanda en el CMV (F. Sánchez, comunicación personal, 28 de junio de 2019).

Sobre los procesos claves del CMV, un $72 \%$ de su personal reconoce que estos se han definido para la consecución del objetivo de la Plataforma MAC. Estos procesos clave, según lo señalado por los responsables desde la SGP-PCM, serían aquellos relacionados a la atención y orientación al ciudadano por parte del personal (F. Vílchez y M. Contreras, comunicación personal, 13 y 25 de junio de 2019).

Por último, los responsables del CMV mencionan la reducción del tiempo de atención, garantizar la calidad del servicio y la correcta atención a la ciudadanía entre los principales objetivos, planes y metas del CMV (F. Sánchez, comunicación personal, 28 de junio de 2019). En adición a esto, el 73 \% del personal del CMV tiene una percepción positiva sobre el reflejo de la Plataforma MAC en los objetivos y las metas del centro; asimismo, se ha comprobado que aquello que identifican como metas y objetivos, como la orientación y ayuda al ciudadano, o la rapidez y la empatía, guarda relación con lo mencionado por los responsables del CVM. A pesar de ello, no se determinó que el personal reconozca planes o metas concretas.

\subsection{Personas}

El plan de gestión de personas tiene la finalidad de reclutar al personal idóneo para cumplir con los objetivos del CMV; sin embargo, este componente no se satisface totalmente en la operación del centro dado que la contratación del personal, al ser realizada de modo independiente por cada entidad participante, no garantiza el cumplimiento del perfil establecido para las labores de orientación y atención al ciudadano.

Por su parte, las actividades de desarrollo que brinda el CMV constan de capacitaciones en procedimientos administrativos y protocolos de atención dirigidos a orientadores y anfitriones contratados por la MDV, sin la participación de asesores de servicio de las entidades participantes. Entonces, aunque no existen políticas ni planes de formación para todo el CMV, el 57 \% del personal encuestado tiene una percepción positiva respecto al estado de las oportunidades de desarrollo. 
En las entrevistas realizadas se identificó la necesidad de una ruptura cualitativa en las capacitaciones que trascienda el componente teórico y evolucione hacia el desarrollo del personal en valores y clima laboral. En ese sentido, se logró definir la orientación al servicio, el manejo de conflictos, la empatía y el compromiso como las competencias críticas del perfil del personal de cara a una atención al público que cumpla con brindar un servicio óptimo.

En cuanto a los medios de reconocimiento y recompensa de los logros del personal a nivel del CMV, el 63 \% de la muestra tiene valoraciones neutrales o negativas. El personal contratado por la MDV afirmó, particularmente, que se reconoce su esfuerzo; sin embargo, no existe evidencia de ningún medio de reconocimiento o recompensa. Al respecto, el único mecanismo de reconocimiento del personal es el Libro de Felicitaciones, donde se les recompensa a través de comentarios positivos de parte de los usuarios del CMV. En este contexto, si bien existen iniciativas de reconocimiento del personal, aún no han sido implementadas.

Los objetivos del personal son establecidos en el Convenio Marco de Cooperación Interinstitucional, suscrito entre la SGP-PCM y la entidad participante. Al respecto, el $53 \%$ del personal encuestado percibe que los objetivos no fueron establecidos de manera realista ni son coherentes con su consecución. A pesar de la valoración negativa respecto al establecimiento de objetivos y metas, estos se cumplen y se evalúan; sin embargo, la percepción negativa del personal se sustenta en su baja participación en la delimitación de objetivos y metas.

Existe una comunicación fluida entre el nivel gerencial e intermedio de la Plataforma MAC, a saber, la SSCAC y el CMV, respectivamente. En contraste, aunque el grueso de la muestra, entre orientadores, anfitriones y asesores de servicio (76 \%), consideró adecuados los medios de comunicación con sus colegas y supervisores, se manifiesta la necesidad de canales de comunicación interinstitucional con el fin de generar mayor coordinación entre asesores de servicio y orientadores.

Por último, es importante mencionar que en cuanto a los documentos de gestión de la Plataforma MAC, aunque el $60 \%$ del personal encuestado percibe que dichos documentos se encuentran a su alcance, es significativo el porcentaje restante (40\%) que considera que la información sobre el CMV no se encuentra disponible.

\subsection{Alianzas y recursos}

Dentro del CMV se ha identificado a los aliados bajo procedimientos establecidos mediante el Decreto Supremo Nº55-2018-PCM, donde se señala que tanto la SSCAC como la MDV pueden aliarse para la implementación y/u operación y mantenimiento del centro MAC. Se comprobó, además, que existe un consenso sobre el beneficio de las alianzas para el CMV. Tanto la SSCAC como los responsables del CMV y su personal consideran que las alianzas establecidas han beneficiado al servicio, pero también señalan que las entidades participantes deben cumplir con brindar el personal necesario y adecuado a fin de que esto no afecte de manera negativa al servicio que se viene brindando.

Por otro lado, con respecto a los recursos económicos, la MDV brinda los recursos necesarios al $\mathrm{CMV}$, ya que —-mediante convenio- esa es su responsabilidad y 
para ello realizan un presupuesto anual; no obstante, los responsables del CMV identifican principalmente las necesidades propias, como la suficiencia de espacio y la disponibilidad de los recursos físicos, las cuales deben ser atendidas por la MDV.

Asimismo, se ha podido determinar que en el CMV se identifican nuevas tecnologías, ya que la coordinadora considera importante la automatización de procesos y contar con herramientas digitales. Es así como, por ejemplo, se actualizó el sistema de colas (QueueSmart) e implementó la Plataforma Virtual Multiservicio del Registro Nacional de Identificación y Estado Civil (Reniec), a la cual puede acceder el ciudadano que asiste al CMV. En ese sentido, estas iniciativas podrían explicar que el $66 \%$ del personal tenga una percepción positiva del uso de nuevas tecnologías en el centro.

Finalmente, con respecto a la gestión de la información y el conocimiento, por un lado, se detectó que el CMV proporciona información del servicio a la SSCAC y a las entidades participantes, pero principalmente cuando se la solicitan, de forma tal que los medios que se ponen a disposición para el intercambio de información no son sistematizados y, por el contrario, este se da a través de correos o por comunicación directa, lo cual no sería solo un problema exclusivo del CMV, sino del Estado en general. Por otro lado, en el CMV, tanto la MDV como las entidades participantes cuentan con mecanismos de seguridad de información, pero propios de cada entidad. Así, aunque en los antiguos manuales de los centros MAC se menciona que el especialista TIC es el responsable de garantizar la seguridad de la información, no se ha encontrado evidencia actual de que se estén realizando dichas acciones.

\subsection{Procesos}

Como se mencionó, existen cuatro procesos clave bajo los cuales el CMV organiza su funcionamiento, los cuales conforman un ciclo de mejora continua en las operaciones del CMV. El diseño y desarrollo de los procesos clave se establecen en función de las necesidades y expectativas de los usuarios del CMV a través de estudios de oferta y demanda, y de encuestas de satisfacción ciudadana.

La gestión por procesos del CMV contiene un conjunto de métricas que comprende el sistema de seguimiento y medición, compuesto por 21 indicadores relacionados con los procesos claves; sin embargo, el CMV se ha enfocado en el seguimiento y la medición del tiempo de espera por trámite. Entonces, si bien la SSCAC diseñó un sistema de gestión por procesos, este aún no fue implementado en el CMV.

En línea con el objeto de incorporar un ciclo de mejora continua a partir de la gestión por procesos, la identificación de oportunidades de mejora se ha centralizado en el nivel gerencial (con la SSCAC de la SGP-PCM, encargada de la formulación y supervisión del cumplimiento de lineamientos y directivas de la Plataforma MAC) por medio de la participación del nivel intermedio del CMV (la coordinadora del CMV, responsable del óptimo funcionamiento del mismo); mientras que los orientadores, anfitriones y asesores de servicio no cuentan con un espacio formalizado para la identificación y proposición de mejoras dentro del horario laboral.

Las medidas de percepción de los usuarios se obtienen de las encuestas de satisfacción ciudadana, el Libro de Reclamaciones y el Libro de Felicitaciones, los cuales 
constituyen insumos para la toma de decisiones para la mejora del CMV. Respecto a estos últimos, aunque la gestión de reclamos y felicitaciones es un proceso crítico de la operación del CMV, el Libro de Reclamaciones y el Libro de Felicitaciones cuentan con una baja participación ciudadana.

En este contexto, aunque el $63 \%$ del personal encuestado valora positivamente las labores del CMV para recopilar las sugerencias, reclamos y encuestas de los ciudadanos, existe una pérdida de interés por conocer la cantidad y el tipo de reclamos recibidos al no formar parte de sus funciones de modo directo; y tampoco existe un espacio formalizado para el planteo de mejoras que intenten dar solución a los reclamos y sugerencias, restándole así importancia a la gestión de reclamos.

\section{Discusión}

Como se mencionó, de la aplicación del Modelo EFQM se obtuvo un diagnóstico de la operación del CMV en términos de los agentes facilitadores del modelo (liderazgo, estrategia, personas, alianzas y recursos, y procesos). Así, el CMV se configura como un espacio donde confluyen entidades públicas de distintos niveles de gobierno y tiene como objetivo prestar una serie de servicios, garantizando la mejor atención al ciudadano.

Dada la complejidad de su arreglo organizacional, se identificó que, aunque la misión, visión, cultura y los valores no han sido desarrollados claramente, el liderazgo de los responsables del CMV se alinea con la finalidad de los centros MAC, la cual se orienta hacia el ciudadano y coloca el énfasis en la calidad. Sin embargo, el accionar para el involucramiento del personal del CMV y actores como las entidades participantes, ya sea en el planteo de mejoras, la difusión de cambios eventuales en el servicio o la identificación de necesidades, sería aún insuficiente.

Ahora bien, se verificó que la estrategia del CMV es coherente con la misión y visión de la MDV y la PCM de brindar servicios de calidad y con un enfoque al ciudadano; y, además, se comprobó el potencial y la idoneidad del servicio en el distrito de Ventanilla. A pesar de esto, la información que se recopila del entorno y las experiencias que se toman para su mejora no serían suficientes. Del mismo modo, la imposibilidad de que el personal identifique planes y metas que incorporen y/o definan los procesos clave denota la necesidad de los mismos con la finalidad de incidir de manera efectiva sobre la atención ciudadana.

En cuanto al personal, se encontró que la gestión del personal del CMV es limitada, dado que se realiza de modo independiente por cada entidad participante. En esta misma línea, no se observaron políticas ni planes de formación de personal en el CMV; es decir, se evidencia el tratamiento desigual en la gestión del personal en las actividades de reclutamiento y desarrollo entre el personal MAC contratado por la MDV y las entidades participantes, lo cual se explica a partir de la imposibilidad de compartir gastos entre entidades públicas y puede incidir negativamente sobre la prestación del servicio, desgastando consecuentemente el clima laboral.

Si bien la existencia del CMV encuentra sentido en las alianzas establecidas entre la SGP-PCM, la MDV y las entidades participantes, es evidente la falta de articulación 
—no solo a nivel de centros MAC, sino del Estado- a través de canales sistematizados y oportunos mediante los cuales se pueda intercambiar información.

Como se mencionó anteriormente, la Plataforma MAC gestiona el componente de calidad con base en la norma ISO 9001:2008 y la SSCAC, por su parte, ha diseñado un sistema de gestión por procesos que en el CMV aún no se ha implementado, lo que evidencia un sistema de gestión elemental enfocado en el control de la calidad -a través de la ISO_ y de naturaleza reactiva. No obstante, los responsables del CMV afirmaron que los esfuerzos para la implementación de la gestión por procesos son parte de un cambio gradual correspondiente a un periodo de transición.

A partir del desarrollo de la presente investigación, surgieron líneas de investigación para el mediano y largo plazo con la finalidad de brindar un mejor entendimiento de la calidad en plataformas de atención al ciudadano, dado que en el Perú no se cuenta con literatura suficiente sobre diagnósticos o evaluaciones en torno al componente de calidad en dichos espacios de atención.

En ese sentido, en el mediano plazo, de cara a las futuras investigaciones del componente de calidad de los centros MAC, existe la necesidad de un estudio de su nivel de madurez previo a la aplicación de modelos más ambiciosos de evaluación de la calidad. A largo plazo, y puesto que la estrategia de la investigación de estudio de caso único se ha visto limitado debido a la naturaleza compleja del CMV, se propone el desarrollo de un estudio de caso múltiple que recoja la complejidad del arreglo organizacional de los centros MAC, ya que su proceso de operacionalización involucra la interacción de entidades de distintos niveles de gobierno.

\section{Conclusiones}

El objetivo de esta investigación fue caracterizar la gestión de la operación del CMV a través de los componentes contemplados en el Modelo EFQM de Excelencia. Sin embargo, aunque el alcance de la investigación se enfoca en la gestión de la operación del CMV, fue indispensable abordar los otros tres procesos clave sobre los que se sustenta el funcionamiento del centro en respuesta a la interrelación entre dichos procesos.

La Plataforma MAC incorpora el componente de calidad basándose en la Norma ISO 9001:2008, que corresponde a la fase de control de calidad que verifica el cumplimiento de los estándares establecidos en la Norma; no obstante, es aún una perspectiva incompleta e insuficiente para configurar un servicio de calidad que satisfaga a los usuarios.

De la aplicación del Modelo EFQM, se puede afirmar que su naturaleza es lo suficientemente robusta como para realizar una caracterización del CMV; sin embargo, el uso del mismo permite recoger una serie de características relacionadas a los agentes facilitadores señalados dentro del Modelo que no pudieron ser identificadas de manera suficiente a causa del sistema de gestión primario del sujeto de estudio. Sin perjuicio de ello, la aplicación del Modelo EFQM para la caracterización de una plataforma de atención al ciudadano es factible.

Finalmente, a partir de los hallazgos obtenidos en la presente investigación, se pueden plantear posibles puntos de mejora para el CMV. Estos podrían estar orientados a reforzar la gestión y participación del personal, haciendo énfasis en aquellos que pertenecen 
a las entidades participantes. Lo mismo sucedería con la búsqueda de una mayor articulación para identificar necesidades y medios para el intercambio de información con las entidades participantes y entre entidades responsables del CMV. Por último, se recomienda asegurar la implementación de una gestión por procesos que utilice indicadores para el seguimiento y la medición de los procesos claves. 


\section{bibliografía}

Alza, C.

2012

Gestión pública: un tema pendiente en la investigación académica. En C. Alza (ed.), Gestión pública: balance y perspectivas (pp. 219-236). Lima: Fondo Editorial PUCP.

\section{Barzelay, M.}

La Nueva Gestión Pública. Un acercamiento a la investigación y al debate de las políticas (trad. A. Dilon). México, D. F.: Fondo de Cultura Económica.

\section{Bresser-Pereira, L.}

La restricción económica y la democrática.

En L. Bresser-Pereira, N. Cunill, L. Garnier,

O. Oszlak y A. Przeworski (eds.), Política y gestión pública (pp. 13-42). Buenos Aires:

Fondo de Cultura Económica de Argentina y CLAD.

\section{Camarasa, J.}

2004

La calidad en la Administración Pública.

Educar en el 2000: revista de formación del profesorado Murcia, (8), 9-20. https://www. researchgate.net/publication/39210040_

La_calidad_de_la_Administracion_publica

Camisón, C., Cruz, S., \&

\section{González, T.}

Gestión de la calidad: conceptos,

enfoques, modelos y sistemas. Madrid:

Pearson Educación S.A.

\section{Centro Latinoamericano}

de Administración para el

Desarrollo (CLAD)

Carta Iberoamericana de Calidad en la Gestión Pública. San Salvador: CLAD.

\section{Christensen, T., \& Laegreld, P.}

Review, 67(6), 1059-1066. https://doi. org/10.1111/j.1540-6210.2007.00797.x

\section{Congreso de la República}

Ley N²7658. Ley de Modernización del Estado. http://www2.congreso. gob.pe/sicr/cendocbib/con4_uibd.nsf/ BCE7AB2E6434B55305257B890053 B271/\$FILE/02A08.pdf (consultado el 28 de octubre de 2018).

\section{Dunleavy, P., Margetts, H., Bastow, S. \& Tinkler, J.}

New public management is dead. Long live digital-era governance. https://www. semanticscholar.org/paper/New-PublicManagement-Is-Dead-\%E2\%80\%94-LongLive-Dunleavy-Margetts/03f502d8044aaaf7 20d5fb327b54c284feb012f2

\section{European Foundation for Quality Management}

Modelo EFQM de Excelencia. https://www. efqm.org/index.php/efqm-model-2013/ download-your-free-copy/

\section{González, F.}

2009

La Administración pública y calidad en el ámbito público. La evaluación de la calidad de los servicios públicos. Normas ISO y modelos de autoevaluación EFQM.

En Documentación sobre gerencia pública, del subgrupo A1, Cuerpo Superior, especialidad de Administración General, de la Administración de la Junta de Comunidades de Castilla-La Mancha. Castilla-La Mancha: Escuela de Administración Regional.

\section{Ipsos Perú}


Jacobs, B., \& Suckling, S.

2007

Assessing customer focus using

the EFQM Excellence Model: a local

government case. The Total Quality

Management Magazine, 19(4), 368-378.

https://www.emeraldinsight.com/doi/

abs/10.1108/09544780710756250

\section{Junta de Andalucía}

Organización Institucional. https://www. juntadeandalucia.es/organismos/sobrejunta/organizacion.html

\section{Montoya, M.}

Reforma del Estado y modernización de la Administración Pública. En Asociación Civil Presidente Ramón Castilla, Memoria de la Primera Conferencia Anual de la Asociación Civil Presidente Ramón Castilla. Reforma del Estado y Modernización de la Administración Pública (vol. I, 227-256). Lima: Fondo Editorial de la Universidad de San Martín de Porres.

\section{Municipalidad Distrital de} Ventanilla

Boletín Anual Centro MAC Ventanilla -

2014. http://www.mac.pe/descargas/

Boletin_2014_Centro_MAC_Ventanilla.pdf

Organización para la

Cooperación y el Desarrollo Económico (OCDE), Banco de Desarrollo de América Latina (CAF), \& Comisión Económica para América Latina y el Caribe (Cepal)

Perspectivas económicas de América

Latina 2018: Repensando las instituciones para el desarrollo. París: Éditions OCDE.
Olías de Lima, B.

2001

La nueva gestión pública. Madrid: Pearson Education.

Patapas, A., Raipa, A., \& Smalsky, V.

2014

New Public Governance: The Tracks

of Changes. International Journal of Business and Social Research, 4(5), 25-32. https://www.researchgate.net/ publication/272625263_New_Public_ Governance_The_Tracks_of_Changes

Ponce, M., \& Pasco, M.

2015 Guía de Investigación en Gestión. Lima: Pontificia Universidad Católica del Perú.

\section{Presidencia del Consejo de Ministros (PCM)}

2013a Decreto Supremo Nº 004-2013- PCM.

Aprueba la Política Nacional de

Modernización de la Gestión Pública.

\section{Presidencia del Consejo de} Ministros (PCM)

Resolución Ministerial Nº48-2013-

PCM. Aprueban el Plan Nacional de

Simplificación Administrativa 2013-2016.

\section{Presidencia del Consejo de} Ministros (PCM)

2018

Decreto Supremo Nº55-2018-

PCM. Decreto Supremo que aprueba disposiciones de la Estrategia de Mejor Atención al Ciudadano - MAC, y sus canales de atención.

\section{Presidencia del Consejo de} Ministros (PCM) 


\section{bibliografía}

Legislativo que aprueba medidas para

el fortalecimiento e implementación de servicios integrados y servicios y espacios compartidos.

\section{Prieto, T. A.}

Elementos a tomar en cuenta para la

implementación de la política de mejor atención al ciudadano a nivel nacional (tesis de maestría, Pontificia Universidad Católica del Perú). http://tesis.pucp.edu.pe/ repositorio/handle/20.500.12404/4873

\section{Robinson, M.}

From Old Public Administration to the

New Public Service. Implications for

Public Sector Reform in Developing

Countries. https://www.undp.org/content/ dam/undp/library/capacity-development/

English/Singapore\%20Centre/PS-Reform_

Summary.pdf

\section{Ruiz-Moreno, A. \& Cuerva, V., \&} Cabeza, D.

Modelo EFQM y organismo autónomo de entidad local: ¿compatibilidad? Cuadernos de Estudios Empresariales, 26, 33-60. http://revistas.ucm.es/index.php/CESE/ article/view/54157/49531

\section{Secretaría de Gestión Pública} de la Presidencia del Consejo de Ministros (SGP-PCM)

Resolución de Secretaría de Gestión

Pública Nº 004-2012-PCM-SGP. Aprueban

Manual del Centro de Mejor Atención al

Ciudadano - Centro MAC.

\section{Secretaría de Gestión Pública de la Presidencia del Consejo de Ministros (SGP-PCM)}

Manual de Funcionamiento de los Centros de Mejor Atención al Ciudadano.

Saiz, J., \& Olalla, B.

Modelo EFQM y creación de valor: una aproximación teórica. Madrid: Universidad Antonio de Nebrija.

\section{Saunders, M., Lewis, P., \&} Thornhill, A.

Research methods for business students (5ta ed.). Harlow: Pearson.

Tejedor, F., Tejedor, G., Muñoz, A., González, R., Portela, J., \& Pastor, A.
Relaciones causales en el Modelo Europeo de Excelencia. DYNA - Ingeniería e Industria, 86(6), 656-661. https://doi. org/10.6036/4088

Torrubiano, J., Fernández, D., \& Gonzáles, D.
Modelos de Evaluación para la
Administración Local. Madrid: Agencia
Estatal de Evaluación de las Políticas
Públicas y la Calidad de los Servicios,
Ministerio de Política Territorial y
Administración Pública.

\section{Vásquez, J.}

Modelos de Calidad en la Gestión Pública. Revista Nacional de Administración, 5(2), 57-78. https://investiga.uned.ac.cr/revistas/ index.php/rna/article/view/745/651

Fecha de recepción: 31 de julio de 2020 Fecha de aprobación: 26 de mayo de 2021 Fecha de publicación: 1 de setiembre de 2021 


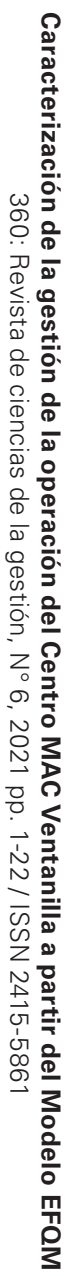

